\title{
DETERMINAÇÃO DA PERMEABILIDADE ABSOLUTA DE UM MEIO POROSO IDEALIZADO ATRAVÉS DO MÉTODO DE LATTICE BOLTZMANN
}

\author{
G. S. SOUZA ${ }^{1}$, J. L. D. ALVES ${ }^{2}$ e R. N. ELIAS ${ }^{3}$ \\ ${ }^{1,2,3}$ Universidade Federal do Rio de Janeiro, Programa de Engenharia Civil, COPPE/UFRJ \\ E-mail para contato: gustavosouza@coc.ufrj.br
}

\begin{abstract}
RESUMO - Propriedades físicas de um meio poroso podem ser inferidas via simulação numérica de um escoamento de fluido realizada na escala dos poros. Neste trabalho, um algoritmo próprio baseado no LBM é utilizado para estimar a permeabilidade absoluta de um meio poroso formado por um arranjo de cilindros dispostos alternadamente. Após comparações com modelos analíticos e outros modelos numéricos, verifica-se a adequação do LBM e do código implementado para solução de problemas de fluxo em qualquer geometria de meio poroso.
\end{abstract}

\section{INTRODUÇÃO}

A caracterização de meios porosos é uma prática bastante comum em algumas ciências aplicadas, tais como geologia, hidrologia, engenharias de petróleo, civil e ambiental. Uma das principais características, a permeabilidade absoluta mede a capacidade de um meio poroso saturado de permitir a passagem de um único fluido devido a uma diferença de pressão imposta. No início era descrita através de equações empíricas como função da porosidade e dimensões das partículas que compõem o meio. Aos poucos, surgiram algumas correlações baseadas na equação de Navier-Stokes para escoamento em meios porosos idealizados (Kuwabara, 1959; Drummond e Tahir, 1984). Atualmente, métodos numéricos são empregados para estimar a permeabilidade do meio sem precisar recorrer a parâmetros empíricos, conforme tem sido mostrado (Sahraoui e Kaviany, 1992; Lee e Yang, 1997).

O Método de Lattice Boltzmann é tratado como uma alternativa ao uso de métodos tradicionais baseados na discretização de equações macroscópicas contínuas na solução de problemas dentro do regime hidrodinâmico. Diferentemente de tais métodos, o LBM é fundamentado na equação de transporte de Boltzmann, formulado em uma escala mesoscópica e possui grande potencial para aplicações envolvendo geometrias e fluidos complexos, sendo por isso amplamente utilizado no estudo de escoamentos em meios porosos e escoamentos multifásicos (Succi, 2001).

\section{METODOLOGIA NUMÉRICA}

\subsection{Equações Governantes}

Um escoamento em meio poroso bidimensional foi simulado na escala de poros utilizando o método de Lattice Boltzmann com a simplificação BGK do operador de colisão. 


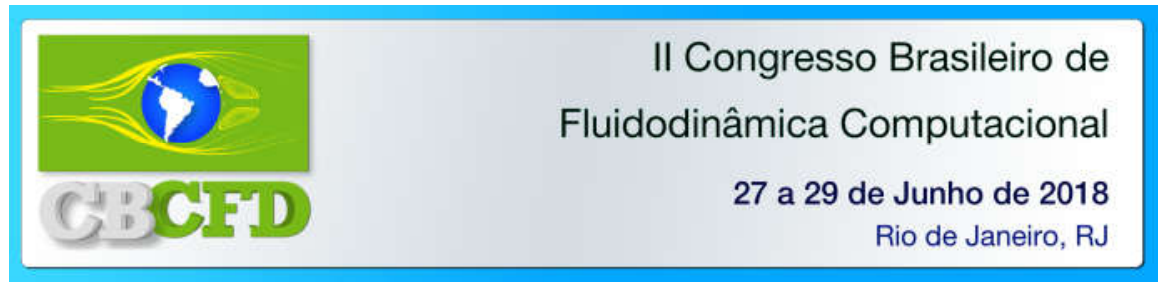

No LBM, o fluido é descrito através da interação entre partículas fictícias que colidem e se movem entre os nós de uma malha cartesiana regular, sendo representados estatisticamente por funções distribuição. A dinâmica do método é governada pela equação de Boltzmann discretizada:

$$
f_{i}\left(x+c_{i} \Delta x, t+\Delta t\right)-f_{i}(x, t)=-\omega\left[f_{i}(x, t)-f_{i}^{e q}(x, t)\right], \quad i=1, \ldots, n
$$

onde o lado direito da equação corresponde ao operador de colisão e $\omega$ é o parâmetro de relaxação temporal, que controla a taxa na qual o problema atinge o equilíbrio. A função distribuição de partículas $f_{i}$ indica o valor esperado do número de partículas com uma velocidade $c_{i}$, em uma posição $x$, em um dado instante de tempo $t$. Grandezas macroscópicas, como densidade e velocidade, e condições de contorno são estabelecidas a partir desta função distribuição de partículas.

Calculou-se o campo de velocidade do escoamento e, em seguida, a porosidade e a permeabilidade absoluta do meio poroso. A porosidade $\varepsilon$ do meio corresponde à razão entre $o$ número de nós fluidos e o número total de nós contidos no domínio analisado. Quanto a permeabilidade, por se tratar de um escoamento laminar, com número de Reynolds pequeno $(R e<1)$, esta pode ser obtida através da relação entre a velocidade média de escoamento nos poros $\bar{u}_{p}$ e o gradiente de pressão. Tal relação é definida pela equação de Darcy,

$$
\begin{aligned}
U_{D} & =\bar{u}_{p} \varepsilon \\
\vec{U}_{D} & =-\frac{\bar{K}}{\mu} \times \vec{\nabla} P
\end{aligned}
$$

\subsection{Descrição do Modelo}

O modelo de meio poroso analisado, conforme mostrado na Figura 1, corresponde a um arranjo de cilindros dispostos alternadamente no espaço. Devido à periodicidade do meio, o domínio considerado na análise foi reduzido a uma célula unitária, de modo a diminuir o custo computacional sem no entanto comprometer os resultados esperados, pois o escoamento na célula resume o comportamento do fluido em toda a extensão do meio poroso.

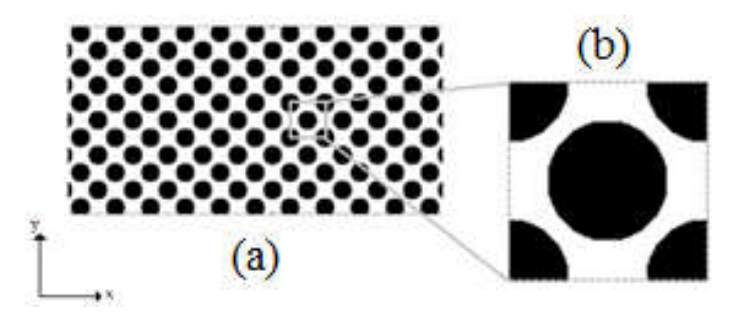

Figura 1 - (a) arranjo de cilindros dispostos alternadamente. (b) célula unitária.

A geometria da célula unitária foi gerada como uma imagem bitmap com resolução idêntica a dimensão do reticulado adotado, de 256 x 256 unidades de lattice, ou um pixel para cada nó do reticulado. Utilizando-se diversas imagens, cada uma com um diferente diâmetro de cilindro, buscou-se variar a porosidade entre $30 \%$ a $95 \%$ (uma imagem a cada aproximadamente 5\%). Esta variação ocorreu obedecendo a recomendação mínima de cinco 


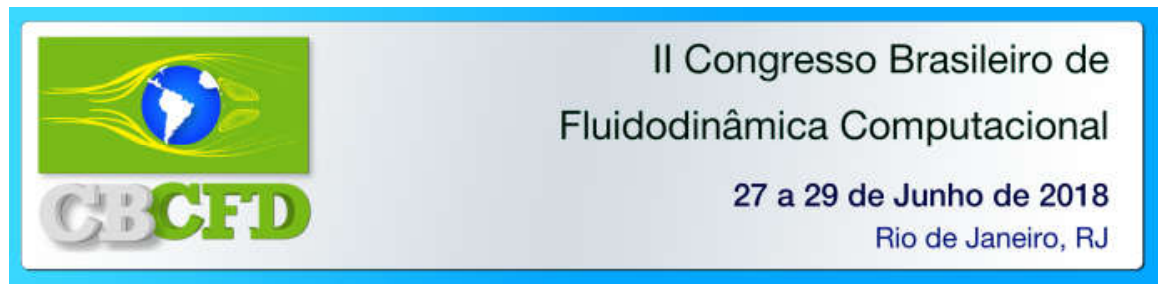

unidades de lattice de espessura de canal (Sukop e Thorne, 2006), necessárias para a reprodução de um correto comportamento hidrodinâmico.

Em seguida, as imagens foram carregadas no processador de imagens ImageJ, sendo então binarizadas e transcritas em arquivos texto. Ao ser executado, o código computacional implementado interpreta as imagens, identificando os pixels de valor " 1 " como integrantes da fase sólida e os pixels de valor " 0 " como integrantes da fase fluida do meio poroso saturado.

A simulação é iniciada com o sistema em equilíbrio. $O$ modelo é inicializado com valores de densidade e velocidade $\rho=1$ e $u x=u y=0$, respectivamente, para cada nó de um reticulado D2Q9. É adotado um parâmetro de relaxação $\omega=1.07$ e são aplicadas condições de contorno periódicas tanto nas fronteiras leste e oeste quanto nas fronteiras norte e sul, além da condição de não-escorregamento do tipo Bounce back nos contornos dos cilindros do meio poroso idealizado. Uma força de corpo igual a $1 \times 10^{-7}$ é imposta na direção $\mathrm{x}$, substituindo o gradiente de pressão necessário para a ocorrência do escoamento. Cabe acrescentar que os valores anteriormente mencionados foram determinados de modo a garantir um escoamento lento através dos poros, no qual os efeitos da inércia são desprezíveis.

\section{RESULTADOS}

Os resultados gerados pelo código implementado na linguagem de programação Fortran foram visualizados através da ferramenta de pós-processamento Paraview. A figura 2 demonstra como o campo de velocidade modifica-se de acordo com a variação dos valores de porosidade ou diâmetro de cilindro.

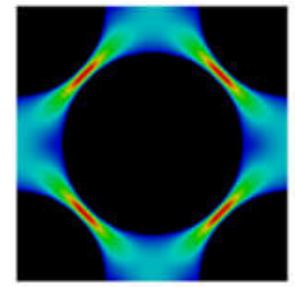

(a)

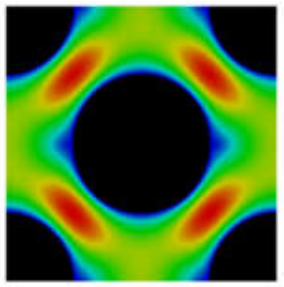

(b)

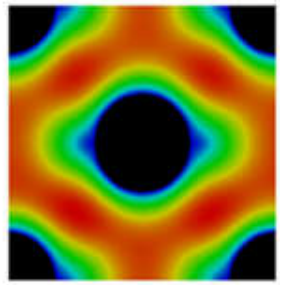

(c)

Figura 2 - Campos de velocidade para: (a) meio poroso com $\varepsilon \cong 30 \%$, (b) meio poroso com $\varepsilon \cong 50 \%$, (c) meio poroso com $\varepsilon \cong 80 \%$. A velocidade do fluido neste caso vai de zero (azul), passando por um valor médio (verde) até atingir a velocidade máxima (vermelho).

A permeabilidade foi normalizada em relação ao tamanho de grão (ou diâmetro do cilindro) e, os valores obtidos para cada percentual de porosidade considerado foram respectivamente comparados com os valores disponibilizados por outros autores na literatura. Analisando a figura 3, foi possível observar que os resultados gerados através do modelo LBM-BGK em geral coincidem com os valores obtidos por meio da correlação proposta por Sahraoui e Kaviany (1992), com exceção dos valores referentes a porosidades fora da faixa de $40 \%$ a $80 \%$, configurados como extrapolações. Os mesmos se aproximam mais dos resultados de Drummond e Tahir (1984) para altas porosidades do que para os de baixas porosidades, um resultado já esperado, pois estes próprios autores reconhecem que o seu modelo é preciso apenas para altas porosidades. Quando comparados aos resultados de Kuwabara (1959), estes também não coincidem em sua totalidade, o que é compreensível pelo fato do método 
proposto por Kuwabara (1959) ser baseado no modelo circular inexato de célula unitária. O modelo simulado está ainda de acordo com o previsto por Lee e Yang (1997).

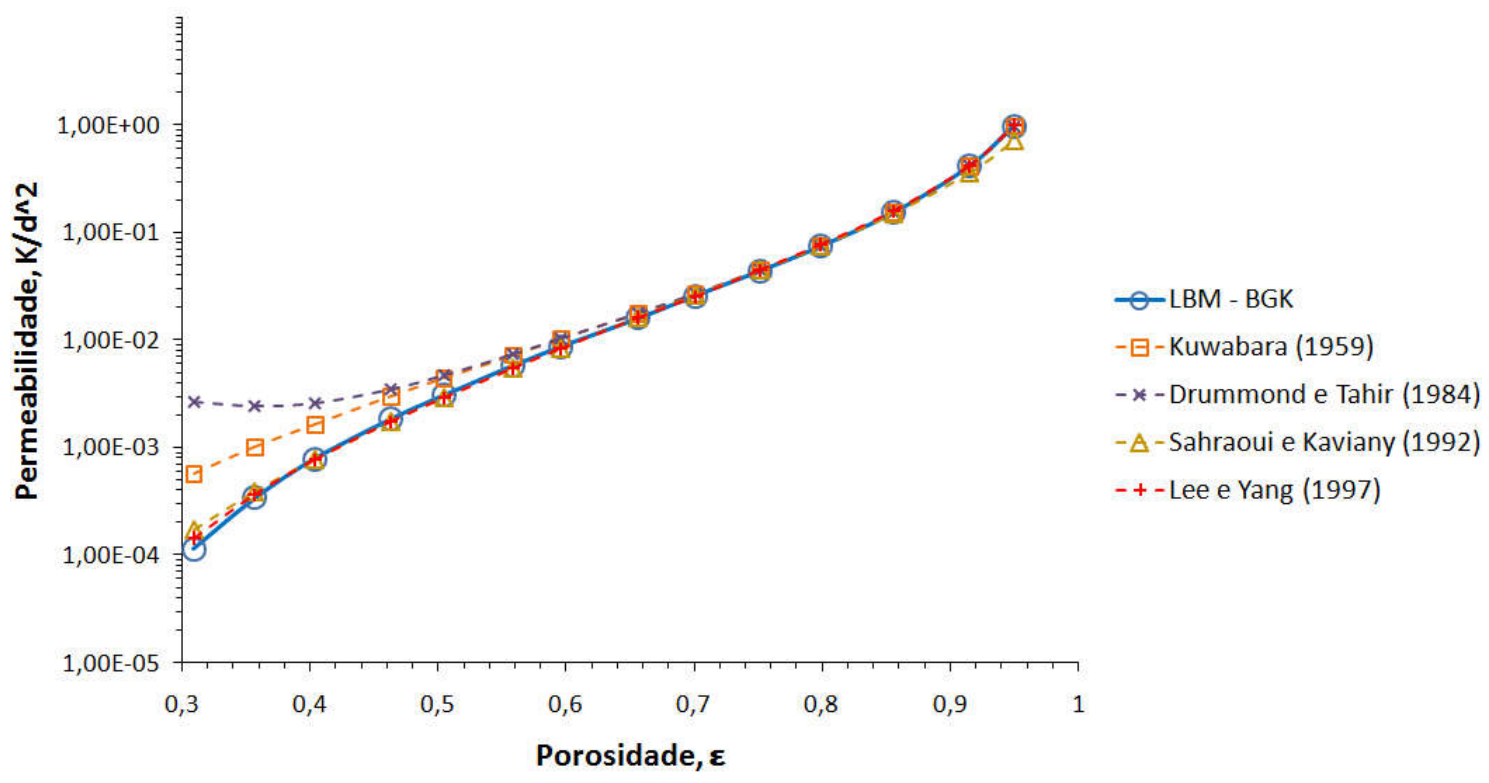

Figura 3 - Resultados de permeabilidade normalizada com o tamanho de grão

\section{CONCLUSÕES}

Com base nos resultados de permeabilidade, pôde-se concluir pela proximidade entre as curvas apresentadas que o método de Lattice Boltzmann é capaz de resolver com sucesso escoamentos em meios porosos. Além disso, a técnica de interpretação de imagens binarizadas contida no código permite a realização de estudos em qualquer estrutura porosa.

\section{REFERÊNCIAS}

DRUMMOND, J.E.; TAHIR, M.I. Laminar viscous flow through regular arrays of parallel solid cylinders. Int. J. Multiph. Flow, v. 10, p. 515-540, 1984.

KUWABARA, S. The forces experienced by randomly distributed parallel circular cylinders or spheres in a viscous flow at small Reynolds numbers. J. Phys. Soc. Jpn., 14, p. 527-532, 1959.

LEE, S.L.; YANG, J.H. Modeling of Darcy-Forchheimer drag for fluid flow across a bank of circular cylinders. Int. J. Heat Mass Trans., 40, p. 3149-3155, 1997.

SAHRAOUI, M.; KAVIANY, M. Slip and no-slip boundary conditions at interface of porous, plain media. Int. J. Heat Mass Trans., 35, p. 927-943, 1992.

SUCCI, S. The lattice Boltzmann equation for fluid dynamics and beyond. Clarendon Press, Oxford, 2001.

SUKOP, M. C.; THORNE JR, D.T. Lattice Boltzmann Modeling - An Introduction for Geoscientists and Engineers. Ed. Springer, 2006. 